\title{
ANÁLISIS DEL PAR ADVERBIAL SOLO-SOLAMENTE COMO MATIZADOR PRAGMÁTICO-DISCURSIVO EN EL ESPAÑOL DE MÉXICO
}

\author{
Lorena Y. MEDINA GómEZ \\ Universidad Autónoma de Querétaro \\ Francisco FERnÁNDEZ GARCía \\ Universidad de Jaén
}

\section{RESUMEN}

En español, el adverbio ha sido estudiado ampliamente en todos los niveles de lengua. No obstante, el análisis de los adverbios en par (adjetivo adverbializado y adverbio con terminación -mente) es reciente. Con el fin de dar continuidad al estudio de los adverbios en par, este análisis da cuenta del comportamiento pragmático-discursivo del par adverbial solo-solamente, pues se observa que, además del significado semántico de unicidad o exclusividad que estos elementos portan, también activan en la enunciación un sentido matizador de tipo atenuación o intensificación en dos distintos niveles de la enunciación, a saber, en el plano semántico y en el plano ilocutivo. En el plano semántico, solo-solamente posiciona al enunciador en relación con alguna parte del significado transmitido por su enunciado; mientras que, en el plano ilocutivo, posiciona al enunciador en relación con algún componente relativo a la fuerza ilocutiva de la enunciación. Así, tanto en uno como en otro se observan enunciaciones que atenúan o que intensifican el contenido proferido por el enunciador. Aunado a esto, se observa que en el plano del significado el hablante induce la activación de interpretaciones valorativas del tipo poco es bueno o poco es malo, y en el plano ilocutivo la matización puede asociarse a la vertiente social del acto comunicativo, es decir, con la cortesía o la descortesía.

PALABRAS CLAVE: solo, solamente, matización, atenuación, intensificación, plano semántico, plano ilocutivo, cortesía, descortesía.

\section{ABSTRACT}

In Spanish, the adverb has been widely studied at all levels of the language. However, the analysis of paired adverbs (i.e., adverbialized adjectives and adverbs with the ending -mente) is recent. In order to further the research of paired 
adverbs, this study analyzes the pragmatic-discursive behavior of the adverbial pair solo-solamente. It has been observed that, in addition to the semantic significance of uniqueness or exclusivity that these elements carry, they also serve as an attenuation or intensification marker in two distinct levels of enunciation: in the semantic field and in the illocutionary act. In the semantic field, the pair solosolamente places the enunciator in relation to some part of meaning transmitted by the statement. Meanwhile, in the illocutionary act, the pair places the enunciator in relation to a component relative to the illocutionary force of the enunciation. Therefore, in both the semantic field and the illocutionary act there are enunciations that either attenuate or intensify the content of the enunciator's utterance. In connection to this, it is observed that in the semantic field, the speaker induces the activation of evaluative interpretations of the type «a little is good» or «a little is bad». Furthermore, in the illocutionary act, the marking may be associated with the social aspect of the communicative act; that is, with politeness or impoliteness.

KEYWORDS: solo, solamente, marking, attenuation, intensification, semantic field, illocutionary act, politeness, impoliteness.

Fecha de recepción: 05/05/2017

Fecha de aceptación: 04/06/2017

Fecha de la versión definitiva: 19/07/2017

\section{INTRODUCCIÓN}

El análisis del adverbio como categoría gramatical en español es extenso y complejo. Ello se debe, para empezar, a las múltiples funciones que estos elementos desempeñan; pero, además, a la dualidad de formas que, con frecuencia, cumplen dichas funciones, como son el adjetivo adverbializado ejemplificado en 1 y el adverbio con sufijo -mente como el del ejemplo $2^{1}$.

(1) El piloto conducía rápido.

(2) El piloto conducía rápidamente.

Entre la amplia gama de análisis se encuentran trabajos de enfoque diacrónico (Kovacci 2000, Kaul de Marlangeon 2002, Hummel 2012); trabajos centrados en el plano morfológico (Egea 1979, Kaul de Marlangeon 2002, Rodríguez 2003); trabajos dedicados al análisis del comportamiento semántico, sintáctico y funcional (Arjona 1991, Rodríguez 2003, Vigueras 1983) y trabajos concentrados en el comportamiento prosódico (Gutiérrez

${ }^{1}$ A partir de este punto, los términos adverbio corto y adverbio largo serán utilizados a lo largo de este análisis para hacer referencia a pares adverbiales como el que se muestra en 1 y en 2 . 
2010, Hoeksema y Zwarts 1991, Medina y Velásquez 2016, Rodríguez 2003, Zubarrieta 2000).

En el caso concreto que nos ocupa, el del par adverbial solo-solamente, además de la dualidad y de la función que desempeña de operador de foco, se detecta en su funcionamiento discursivo un carácter polisémico que hace patente la necesidad de profundizar en su estudio.

En cuanto a su función, en primer lugar, el par adverbial solo-solamente, desde la perspectiva de la estructura informativa, se considera un operador de foco, dado que su tarea principal es la de focalizar para identificar, restringir o excluir a un elemento específico dentro de un conjunto de posibles alternativas (cf. Ferrari et al. 2011: 30-41, Gutiérrez 2008: 378, Gutiérrez 2010: 626-632, Hummel 2012: 145). De este modo, en el ejemplo 3 solo focaliza a Pedro como único ente interviniente en el evento de rendirse; en contraste, en el ejemplo 4 solo focaliza el evento, es decir, rendirse es lo único que hizo Pedro (no corrió, no se defendió: no hizo otra cosa más que darse por vencido).

(3) Solo Pedro se rindió.

(4) Pedro solo se rindió. (Gutiérrez 2008: 378)

Resulta manifiesto, por consiguiente, que la interpretación de ambos ejemplos es diferente debido al alcance focal que solo-solamente tiene sobre las unidades lingüísticas.

A dicha función focalizadora hay que añadir, en segundo lugar y de manera muy relevante, el potencial de los elementos analizados como operadores con valor argumentativo, cuya función es la de orientar el discurso en una dirección determinada, con el fin de facilitar las inferencias que se desean comunicar (cf. Portolés 1993: 158, Anscombre y Ducrot 1994: 191).

Por otro lado, el carácter polisémico que albergan estos elementos adverbiales se observa en la medida en que dichas formas pueden asociarse a significados como el de unicidad, cuando hacen referencia a un único ente en el universo (ejemplificado en 5); o al de restricción o exclusión-dependiendo de la imposición de la perspectiva del enunciador-, cuando limitan, excluyen o especifican un evento o situación (como en 6) (Anscombre y Ducrot 1994: 190, Seco 2002: 417, Martínez 2006: 1438, Gutiérrez 2008: 378, RAE y ASALE 2010: 764, Lara 2011: 1524, Ferrari, Giammatteo y Albano 2011: 30, Fuentes Rodríguez 2009: 323, Hummel 2012: 145, Langacker $1991,2000)^{2}$.

\footnotetext{
${ }^{2}$ Aunque los ejemplos fueron tomados tal cual del corpus, en la parte inicial se proporciona entre corchetes el contexto discursivo del evento para su fácil aprehensión. Acerca del corpus manejado en la investigación, véase el epígrafe 3.
} 
(5) [INFORMANTE EXPLICA EL USO DE LOS SARCÓFAGOS]

I: el sarcófago / que supuestamente está ahí / que lo robaron / el sarcófago siempre permaneció / supuestamente siempre permaneció vacío / nunca tuvo ningún cuerpo // era para los iniciados / ahí los preparaban // los metían varios días y él tenía que despegar / tenía que preguntar / tenía que ponerse en contacto con los ángeles // ya no con dios porque la biblia misma te dice / el que ve a dios / morirá / nadie puede ver / puede ver a dios directamente // solamente Jesucristo que estaba capacitado porque él / él venía de donde / estaba dios / él trabajó junto con dios cuando se creó todo // entonces el ser humano no está capacitado para verlo // y ahí fue donde entendí que podías viajar por medio de la pirámide

(6) [INFORMANTE DICE POR QUÉ ACUDE A MISA]

I: pero / o sea / por ejemplo si voy a misa no es con // o sea como que / a eso / como que

E: a criticar

I: a n / no no / o sea / voy a misa solamente para estar con dios sea es

E: ya

I: o sea / para estar más cerca de dios no / no así de que / ¡ay! voy porque tengo que es / que escuchar al padre no / nada más es para yo / yo estar cerca de dios si

Adicional a los significados anteriores y a las funciones discursivas señaladas más arriba, se observa de manera interesante que el par adverbial solo-solamente ejerce una función estratégica de matización pragmática ${ }^{3}$, de tipo atenuador o intensificador (Gutiérrez 2008: 378, Fuentes Rodríguez 2009: 323-324, RAE y ASALE 2010: 593, Hummel 2012: 145). En este sentido, es importante resaltar que los valores de atenuación o de intensificación no son alternativos al significado básico de unicidad, restricción o exclusión, sino que, cuando se manifiestan, lo hacen de forma paralela, de manera que los valores de ambos planos coexisten en el discurso.

Así podemos observarlo, por ejemplo, en el pasaje 7. Puede apreciarse que solo denota unicidad como valor primario; no obstante, en el evento al que se alude, la entrevistadora minimiza su indiscreción al haber divulgado que la informante se casaría, de manera que dicho valor semántico es únicamente una parte de lo que el adverbio transmite en este contexto: en paralelo a él, surge un valor estratégico de atenuación.

(7) [ENTREVISTADORA E INFORMANTE COMENTAN SOBRE LA BODA DE LA INFORMANTE] I: o sea no es/ ya no es ni conveniencia/ porque// bueno/ no sé/ hasta qué punto se le pueda llamar así/ pero yo conozco igual así los casos contrarios/ ¿no?/ en donde la/ la chava es la// la de la lana/ o sea/ la que trabaja

\footnotetext{
${ }^{3}$ Entendemos aquí la matización pragmática como la potencialidad de desarrollar, en contexto, una carga de significado adicional a la básica, a la codificada, que puede contribuir a modificar el potencial argumentativo del enunciado.
} 


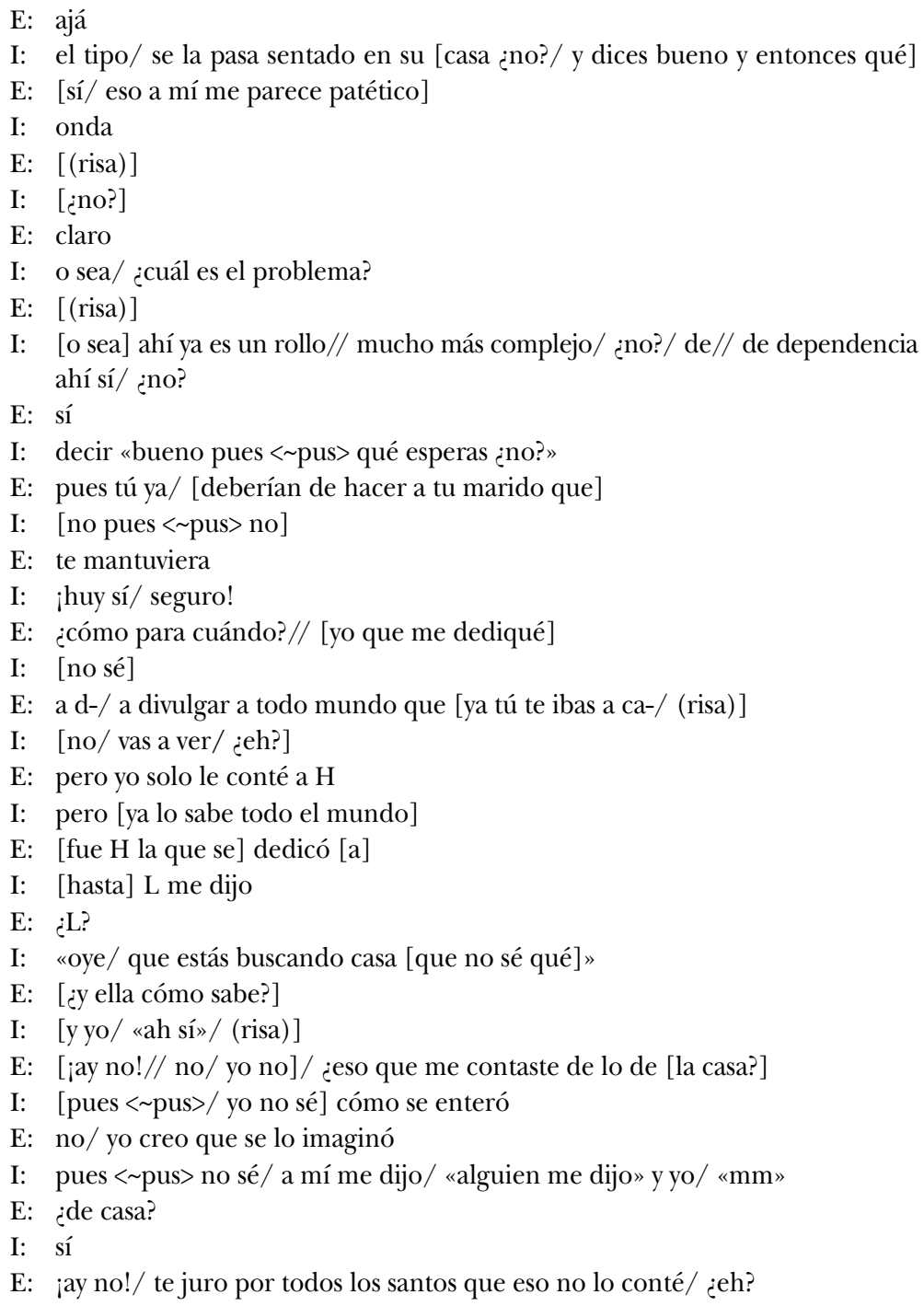

Algo similar sucede en el ejemplo 8. Solo hace prominente el valor primario de restricción pero, en paralelo, se activa un valor estratégico de intensificación.

(8) [INFORMANTE EJEMPLIFICA CON UNA EXPERIENCIA EL EXCESIVO COSTO DE LOS MEDICAMENTOS]

I: [y hay algunos]/y hay algunos que valen/ „¡ciento y pico!!

E: ihh

I: y no creas que traen muchas pastillas 
E: sí/ unas [cuatro/ ¿no? (risa)]

I: [traen dieciséis/ no]/// la habitualmente eh < eh:> la mayoría// mínimo traen diez/// eh < eh:>/ un promedio de dieciséis/ y otras traen veinte exagerando/ no traen más

E: jsí!/ yo el otro día $\mathrm{mm} / /$ no me acuerdo qué me enfermé/ y me recetaron un antibiótico bastante fuerte/ y venían sólo ¡dos pastillas! en el/ en la cajita y costaba trescientos pesos/ la medicina// $\mathrm{mmhh} /$ dije «híjole»

I: sí/// no pues < pus> sí hay medicamentos que te vale doscientos y pico/ una sola ampolleta

$\mathrm{E}:[\mathrm{mh}]$

I: [nada más]/ y párale de contar

E: híjole

Por todo lo anterior y con el objeto de indagar sobre el hallazgo de los valores pragmático-discursivos de atenuación e intensificación que porta el par adverbial solo-solamente, aún insuficientemente analizados por la bibliografía especializada, el presente trabajo se propone avanzar en dicho camino conforme a las directrices que a continuación presentamos.

\section{HiPÓtesis y OBJETIVOS}

Conforme a lo que prevé la bibliografía ${ }^{4}$, la observación sistemática de nuestro corpus hace patente que, en el español de México, el par adverbial solo-solamente permite al hablante añadir un componente pragmático a sus enunciaciones. Así, el hablante, sobre la base del contenido semántico primario transmitido por el adverbio, expresa un componente de significado adicional de carácter estratégico, proyectando cierta información de forma atenuada o intensificada.

Ante tal constatación, el objetivo de este trabajo, desde una perspectiva de análisis pragmático-discursivo, es observar en profundidad el comportamiento de estos elementos como matizadores. Nos proponemos, de este modo, confirmar la presencia del fenómeno y, simultáneamente, determinar en qué circunstancias dicha forma léxica puede ejercer funciones tanto de atenuación como de intensificación.

\section{CORPuS}

El material sobre el que hemos trabajado -así como los ejemplos que aquí presentamos- procede de dos corpus representativos del habla informal de México: el Corpus Sociolingüístico de la Ciudad de México (CSCM)

\footnotetext{
${ }^{4}$ Véase el epígrafe 0.
} 
(Butragueño y Lastra 2011) y el Corpus del Habla de Monterrey (CHDM) (Rodríguez, Flores y Pérez 2012). Ambos corpus pertenecen a un proyecto mayor, denominado «Proyecto para el estudio sociolingüístico del español de España y de América» (PRESSEA), cuyo objetivo es hacer una recopilación de variantes orales del español de diferentes ciudades de España y del continente americano (cf. Rodríguez, Flores y Pérez 2012).

\subsection{Características de los corpus CSCM y CHDM}

Tanto el CSCM como el CHDM representan el habla oral de dos de las ciudades más importantes de la República Mexicana. El primero preserva el habla de la Ciudad de México y el segundo, la de Monterrey. Ambos guardan los mismos estándares y la misma metodología; por ello, en esta investigación se consideran análogos.

Con el fin de obtener una producción espontánea, todas las entrevistas se efectuaron en contextos ordinarios, buscando así crear un ambiente de naturalidad; esto es, los informantes se encontraban en su casa, trabajo o en su universidad o centro de estudios. La mayoría de las entrevistas se realizaron de manera individual; no obstante, algunas sucedieron con algún compañero o familiar. Las grabaciones fluctúan entre los 45 minutos y las dos horas de duración. Los temas que se tratan tienen que ver con la vida diaria, como la niñez, los juegos, los problemas de trabajo, los estudios, el estrés, el dinero o las relaciones personales, entre otros. Los materiales tienen amplia variedad discursiva, ya que incluyen fragmentos narrativos, argumentativos y descriptivos.

Cada corpus consta de 108 entrevistas, número suficiente según la representatividad de la muestra $\geq 0,025$ (cf. Rodríguez, Flores y Pérez 2012). Dichas entrevistas se distribuyen de acuerdo con tres variables sociales: género, edad y grado de instrucción. En cuanto al género, participan igual número de hombres y de mujeres, 54 de cada uno. La variable edad se divide en tres grupos, identificados como generación 1, 2 y 3: la generación 1 involucra participantes de 20 a 34 años; la generación 2, de 35 a 54, y la generación 3, de 55 años en adelante. Por último, de la variable grado de instrucción se crean tres grupos, nivel 1, 2 y 3: en el nivel 1 se ubica a aquellas personas que tienen de 0 a 8 años de instrucción formal, esto es, desde analfabetos hasta educación secundaria incompleta; en el nivel 2, se coloca a aquellas personas con 9 a 14 años de instrucción, es decir, personas que cuentan con certificado de secundaria y licenciatura incompleta o carrera técnica; y, en el nivel 3, a aquellos que tienen 15 años de instrucción formal como mínimo, esto es, personas con grado de licenciatura o más. 
El CSCM fue coordinado por Pedro Martín Butragueño, del Colegio de México, y por Yolanda Lastra, de la Universidad Autónoma de México (Lastra y Butragueño 2000, 2003, 2009; Butragueño y Lastra 2011). Este proyecto se delimita geográficamente a 27 entidades, a saber, 16 delegaciones del Distrito Federal ${ }^{5}$ y 11 municipios conurbados del Estado de México, a los cuales se les denominó Zona Metropolitana de la Ciudad de México (ZMCM).

El CSHM, por su parte, fue coordinado por Lidia Rodríguez Alfano, María Eugenia Flores Treviño y Tzitel Pérez Aguirre, de la Universidad Autónoma de Nuevo León (2010). Este proyecto se delimita geográficamente a 7 municipios del área metropolitana de Monterrey y a la zona conurbada.

\subsection{Recopilación de datos}

Un análisis exhaustivo de los corpus elegidos para nuestra investigación reveló la presencia de 283 ejemplos que contienen solo o solamente, de los que 177 son cortos y 106, largos. Es importante mencionar que únicamente se tomaron en consideración aquellos ejemplos en los que el elemento de análisis apareció de manera individual, y no en construcción como no solo... sino, no solo... también o solo-solamente que, dado que los valores semánticos que se activan son el de 'inclusión' - para el primero y segundo- y el de 'condición' -para el tercero-, usos que quedan fuera de los límites de nuestra investigación.

Por otra parte, conviene insistir en algo que ya adelantábamos al comienzo del trabajo, como es la equivalencia funcional entre solo y solamente. Así, nuestra investigación trabaja indistintamente con ambas formas adverbiales debido a la conmutabilidad que entre ellas existe, es decir, debido al hecho de que solo puede ser sustituido por solamente -y viceversa- en todas las posiciones sintácticas y focalizando, desde la estructura informativa, a cualquier elemento, frase o enunciado de la configuración lingüística.

Es importante comentar que la única posición sintáctica donde solamente no puede conmutarse por solo es aquella en la que la forma larga se encuentra al final de frase o enunciado, como en [ENTERARSE] ver lo que pasaba en la tele solamente vs. * [ENTERARSE] ver lo que pasaba en la tele solo. La razón es que, cuando solamente se encuentra al final de la configuración lingüística o frase, y se sustituye por solo, la semántica adverbial inicial se torna adjetival (sin compañía), lo que desencadena una predicación secundaria depictiva (cf. Himmelmann y Schultze-Berndt 2005). No obstante, con

\footnotetext{
${ }^{5}$ De acuerdo con una reforma constitucional promulgada por el presidente Enrique Peña Nieto, a partir del 29 de enero de 2016 el nombre oficial de la capital del país deja de ser Distrito Federal y cambia por el de Ciudad de México.
} 
esta posición sintáctica únicamente aparecen tres casos de los 283 ejemplos que conforman la base de datos.

\section{SOLO-SOLAMENTE COMO MATIZADOR PRAGMÁTICO-DISCURSIVO}

El lenguaje es la principal herramienta de comunicación entre los seres humanos. Su uso en los distintos propósitos comunicativos está gobernado por reglas sociales y por la cognición humana; tales reglas y entorno cognoscitivo compartido permiten que el usuario acceda a este medio de comunicación y lo utilice con sapiencia y dominio (cf. Mey 1979). Dado que la comunicación es indispensable para la interrelación con otros, y las reglas sociales y conocimiento compartido lo son para mantener dicha interacción, el hablante hace uso de variadas estrategias pragmático-discursivas con el fin de que sus enunciaciones lleguen al destinatario conforme a su propósito, y así, pragmáticamente establecer relación entre los participantes (Anscombre y Ducrot 1994: 113). En este sentido, la matización es una estrategia discursiva de la que se vale el hablante para imprimir en sus enunciaciones juicios de valor o puntos de vista de forma añadida al contenido semántico que transmite. De acuerdo con la intención comunicativa, tal matización puede adquirir de manera relevante dos direcciones: la atenuación y la intensificación. Y estos dos matices pueden ser activados, entre otros mecanismos, mediante el uso del par adverbial solo-solamente ${ }^{6}$.

En 1977, Barrenechea ya hacía alusión al uso de los adverbios como operadores pragmáticos indicadores de actitud oracional, de los cuales comentaba que eran «indicadores de actitud del hablante con respecto al contenido del discurso» (Barrenechea 1977: 313); no obstante, dentro de este análisis, no aparece ningún elemento de nuestro par adverbial. Por otro lado, Anscombre y Ducrot (1994: 189) distinguen tres empleos de solamente (en francés seulement), al que consideran un operador con valor argumentativo, cuyo significado se fundamenta en el enunciado en que se introduce. Así, observan que seulement es empleado como operador de coordinación equivalente a pero; también como operador restrictivo al que puede sustituirse por no más que o no otra cosa más que; asimismo, reconocen a seulement en contextos polarizados, en este caso negativos, con sentido minimizador, sustituible por aunque no fuera más que o aunque no fuera más que por eso.

En relación con estas aportaciones de Anscombre y Ducrot (1994), únicamente el segundo empleo es el que reconocemos por asemejarse a los

\footnotetext{
${ }^{6}$ Nos referimos a las dos direcciones esenciales que el proceso de matización adquiere en los usos de solo-solamente. Por supuesto, tal tipo de añadidos implícitos puede tomar cuerpo, en otros casos, en forma de comentarios colaterales, añadidos modales, etc.
} 
casos que aparecen en nuestro corpus con significado básico, no pragmático; señalemos, además, que en ese estudio solo se hace alusión a la forma larga, seulement, y no a la corta. Por otra parte, es interesante observar que Anscombre y Ducrot observan el sentido minimizador en el tercer empleo; lo hacen, sin embargo, únicamente en referencia al uso de seulement con el adverbio negativo no antepuesto, un tipo de casos que nosotros hemos dejado fuera -según quedó explicado en 2.2- debido a la extensión que implicaría su incorporación.

Por su parte, Kaul de Marlangeon (2002: 133) da cuenta de los adverbios que responden a sentimientos o percepciones del hablante y a su intención pragmática de evaluación o a su deseo de expresar acuerdo o desacuerdo, pero sin aludir a solo-solamente en su análisis. Asimismo, Rodríguez (2003: 36) propone una clasificación exhaustiva de los distintos tipos de adverbios, entre los que distingue a los adverbios con función pragmática, clasificados en dos grupos, a saber: los orientados al hablante y los que remiten a un punto de vista; sin embargo, solo-solamente tampoco aparece dentro de su análisis. Por último, Martínez (2006: 1434-1435) hace mención, de manera general, a la innovación que presentan los diccionarios al añadir información pragmático-discursiva de algunos adverbios. Con ello se hace referencia al punto de vista, a la intercomunicación y al uso que hacen los hablantes de estos elementos lingüísticos en el discurso. Dentro de la lista que ofrece para ejemplificar, solamente aparece como uno de los adverbios que han sido enriquecidos en su descripción semántica, pero no presenta mayor detalle.

De este modo, la bibliografía especializada que alude de una u otra forma a adverbios con funciones pragmáticas de tipo discursivo no profundiza, en general, en el funcionamiento de solo-solamente -Martínez (2006) lo enlista, pero no va más allá, y Anscombre y Ducrot (1994), que entran más en materia, únicamente dan cuenta de la forma larga. Además, el resto de los autores atribuyen esta cualidad a los adverbios largos, que ellos observan, no así a los cortos.

En fechas más recientes, Hummel (2012: 145) -partiendo de Hartmann (1999: 29) - reconoce al adverbio corto solo como un diminisher (es decir, como un 'atenuador'); asimismo, identifica con función de enfoque e intensificación al adverbio largo solamente (2012: 262). Sin embargo, más adelante mostraremos que tanto el adverbio corto como el largo pueden actuar como atenuador o como intensificador, sin que tales valores sean privativos de una u otra forma.

Vayamos, pues, conforme a los objetivos marcados en el epígrafe 1, al análisis del potencial matizador -atenuador e intensificador- del par adverbial solo-solamente y de la configuración que dicha matización puede adoptar dentro del enunciado. 


\subsection{Atenuación vs. intensificación}

Conforme a Briz (2012: 37), la atenuación es un hecho lingüístico y pragmático que «responde a un conjunto de recursos verbales y no verbales con los que se minimiza estratégicamente» el impacto comunicativo de un enunciado. Dicho conjunto de recursos abarca, en lo verbal, tanto el significado explícito como el implícito; y, en lo no verbal, un variado abanico de mecanismos como exclamaciones, risas, entonaciones, etc.

A nivel pragmático, la atenuación busca menguar el posible efecto negativo de lo dicho y resguardar la imagen del que escucha (cfr. Cestero, Albelda y Briz 2014: 296, Cestero y Rodríguez 2014: 2). De este modo, permite que tal efecto negativo no incomode, hiera o moleste al interlocutor. No obstante, con la atenuación no solo se busca que el mensaje llegue de forma sutil, sino que también, y al mismo tiempo, que la emisión de dicho mensaje no sea percibida como proferida violentamente por el hablante. Así las cosas, la atenuación previene o repara el posible efecto negativo de lo expresado de manera bidireccional.

La intensificación, por el contrario, busca un efecto comunicativo opuesto, a saber, el de magnificar el valor expresivo de lo comunicado. De este modo, cuando se intensifican contenidos considerados negativos ${ }^{7}$, el hablante no pretende menguar las posibles consecuencias negativas de lo dicho ni salvaguardar la imagen de su interlocutor, sino todo lo contrario: da a conocer su disconformidad, desacuerdo o inaceptabilidad sobre algo.

En la medida en que la intención de la intensificación pueda ser, pongamos por caso, evidenciar una situación con la que no se está conforme, se otorga énfasis al discurso, lo que permite que tales contenidos adquieran mayor relevancia en la interacción; por esta vía, puede buscarse que el interlocutor alcance el significado que el emisor pretende y coincida con él en la gravedad de la situación. Así, la empatía en lugar de la imagen es lo que el emisor procura; no obstante, es un hecho que, si se gana la empatía, la imagen quedará, probablemente, salvaguardada ${ }^{8}$.

Pues bien, se ha observado que tanto solo como solamente pueden cumplir una función matizadora en ambos sentidos, ya sea que atenúen, ya sea

${ }^{7}$ Por supuesto, este no tiene por qué ser necesariamente el caso. También pueden intensificarse contenidos interaccionalmente positivos.

8 Debe quedar claro, no obstante (y volveremos sobre ello al final de este trabajo), que el plano de la atenuación y la intensificación es el de los mecanismos lingüísticos (y no lingüísticos), vacíos en sí mismos de contenido funcional, diferente del plano psicosocial, en el que actúan la imagen social y las fuerzas de (des)cortesía con ella relacionadas. Dicha diferencia es la que fundamenta la distinción, a la hora de analizar el funcionamiento de la (des)cortesía, entre mecanismos y estrategias funcionales, analizada en trabajos como Fernández García 2015 y 2016. 
que intensifiquen una enunciación. Y el cumplimiento de esa doble función no constituye, desde luego, un hecho excepcional. Cestero y Rodríguez (2014: 4), por ejemplo, comentan cómo cierto mecanismo lingüístico (en este caso, morfológico) puede actuar como atenuador o intensificador de acuerdo a las características discursivas y contextuales presentes:

[...] el diminutivo que se supondría atenuador por excelencia en cuanto disminuye la dimensión del referente, en ocasiones se emplea para denotar cariño y, por el contrario, puede usarse para insultar a alguien (esto es en la mayoría de los casos en que se diminutiza un apellido como al llamar a alguien «Gutierritos», y, sin embargo, en Monterrey se llama Gonzalitos en señal de reconocimiento, admiración y aceptación amorosa al primer médico de la ciudad, y quien dedicó su vida a salvar vidas sin exigir la remuneración económica que la labor presupone).

\subsection{Matización semántica vs. matización ilocutiva}

Por otro lado, hemos de reparar en el hecho de que limitarnos a distinguir la matización atenuadora de la intensificadora en los usos de solosolamente introduciría una equívoca heterogeneidad dentro de tales categorías. Ello se debe a que, al margen de la dirección que adopte la matización (atenuadora o intensificadora), esta puede tomar cuerpo en dos distintos niveles de la enunciación: el plano semántico y el plano ilocutivo. Introduciremos, por consiguiente, esa nueva distinción, y hablaremos de atenuación y de intensificación en relación con cada uno de tales niveles del enunciado. De este modo, solo-solamente matiza, en el primer caso, posicionando al enunciador en relación con alguna parte del significado transmitido por su enunciado, mientras que, en el segundo, lo hará posicionándolo en relación con algún componente relativo a la fuerza ilocutiva de la enunciación.

En ambos tipos de matización, además de la presencia de solo-solamente, aparecen otros tipos de reforzadores pragmáticos, como el uso de interjecciones, la presencia del pronombre personal de primera persona, la repetición de palabras o incluso frases, exclamaciones, uso de diminutivos, preguntas, risas, información contextual relevante o de palabras con alto contenido semántico y cultural, así como cambios prosódicos (entonaciones altas o bajas, alargamientos o acortamientos). Adicionalmente a las anteriores, Cestero et al. (2014) reconocen también el uso de elipsis e impersonalizaciones, entre otros mecanismos.

En este sentido, es importante hacer notar que, aunque aparezcan en el discurso otros elementos que apoyen la matización, la presencia de solosolamente resulta indispensable para guiar la interpretación, pues si este se 
elide la atenuación o la intensificación se pierden, y lo que queda es una narración de los hechos. Esta es la razón y la evidencia por la que, pragmáticamente, hemos de sostener que solo-solamente actúa, en un significativo porcentaje de sus apariciones, como matizador.

\section{ANÁlisis de ejEMPLOS}

En función de las distinciones establecidas en los epígrafes 3.1 y 3.2, y tras un meticuloso análisis del corpus, se ha hallado la presencia de 85 ejemplos (56 cortos y 29 largos) en los que, añadida al sentido base del adverbio, podemos hallar alguna de las variantes de matización pragmática; en el resto, 198 casos, solo está presente el sentido base.

\subsection{Matización semántica}

Como explicábamos en 3.2, el primero de los ámbitos en que solo-solamente puede actuar como elemento matizador es el plano del significado. Mediante este uso estratégico, el operador orienta el contenido de manera tal que un evento o su resultado sean percibidos como poco importantes (atenuación), o todo lo contrario, muy relevantes (intensificación). Así, a través de estas lecturas, el hablante induce la activación de interpretaciones valorativas del tipo poco es bueno o poco es malo. Es decir, más allá del valor objetivo del significado de lo poco (pongamos por caso, en un ejemplo descontextualizado, solo son dos), la aparición de nuestro adverbio en determinadas situaciones comunicativas actúa como detonante del surgimiento de efectos contextuales específicos que valoran ese «ser poco» como algo bueno o algo malo.

\subsubsection{Atenuación semántica}

Podríamos pensar que la función atenuadora es la más natural a solosolamente, dado su contenido semántico. De este modo, cuando se expresa algún significado en el que lo mucho es malo, la presencia de nuestro par adverbial activa un valor escalar interpretado como poco es bueno, lo que remite a una escala y, de esa escala, a la parte baja. Para lograr esta conceptualización escalar, el operador, con ayuda del foco y del contexto, guía la interpretación del discurso.

En el ejemplo 9, el hablante minimiza e incluso hace parecer insignificante el hecho de que uno de sus compañeros haya salido lastimado en una pelea callejera. En este caso, solo funciona como un operador anti- 
orientado (Escandell 1993: 120, Anscombre y Ducrot 1994: 191, Fuentes Rodríguez y Alcaide Lara 2007: 26), dado que guía y atenúa la carga semántica, el peso de lo dicho, de manera que lo malo, que se esperaba que fuera mucho, al final es poco, y eso es bueno. De este modo, la contraexpectativa desempeña un papel fundamental en este tipo de matización, dado que, en este caso, pelearse con alguien mayor implicaría una desventaja; y, pese a eso, salimos airosos, ganosos, ilesos. Así, el elemento antiorientado da la vuelta al planteamiento, rompiendo con las expectativas.

De manera adicional, el hablante proporciona información contextual relevante al interlocutor; esto es, da a conocer las condiciones en las que se llevó a cabo el evento. Además, utiliza palabras de alto contenido léxico y cultural, como echarme porras, caguamazos o gracias a dios, todo ello necesario para que el oyente conozca los pormenores del evento y, de esta manera, pueda lograrse la empatía y afinidad respecto de la situación.

(9) [INFORMANTE RECUENTA UNA PELEA CALLEJERA]

I: me tocó a mi peliarme con un chavo así / solo / este / digo / no es por echarme porras <observación_complementaria = 'adularse' $/>$ pero / sí le / sí lo llegué a descontar dos tres veces / a mí me descontaron ;fun! así a campal / gracias a dios a todo dar/ no no hubo

E: no hubo / de que

I: de balazos

E: caguamazos <observación_complementaria $=$ 'golpes con botellas de caguama o envases grandes de cerveza'/>

I: gracias a dios no fue / fue

$\mathrm{E}$ : es que te puedes hasta morir ¿no? / de un

I: fue limpio / fue limpio el / el tiro ahí / eso sí sí / gracias a dios creo que salimos airosos / este / y sí me tocó peliarme con uno / con un chavo / más grande que yo / yo como / que tenía ¿qué será? / dieciséis diecisiete años / el chavo tenía como veinte

E: mjm

I: y me / gracias a dios él no me pegó yo / que nos peliamos yo y él y / pero llegó un chavo por atrás y me descontó o sea / en sí no le pegué / él no

E: mjm

I: y yo / como te digo / yo creo que salimos ilesos o sea / salimos ganosos ¿verdad?

E: mjm

I: de ellos sí dejamos a dos tres / un poco golpiados

E: sí

I: y ellos nomás un / amigo de nosotros porque sólo se resbaló y se cortó contra la / contra la / contra el suelo

E: mjm

En el ejemplo 10, por su parte, el informante narra una experiencia en la que estuvo en peligro de muerte. A través del uso de solo revela que, de 
acuerdo con su percepción, el herirse los pies fue algo insignificante en comparación con lo que pudo haber sufrido, pues, según explica, caían muros enteros, escombro, vidrios y habia que saltar descalzo; de hecho, resultan muy reveladores en este sentido la apertura de la cláusula con el adverbio afortunadamente y el uso del diminutivo poquito. Es decir, ante la expectativa de lo mucho (malo) que podría haber ocurrido, de nuevo se evalúa atenuadoramente, introduciendo ese elemento antiorientado en que lo poco es bueno.

(10) [INFORMANTE CUENTA SU EXPERIENCIA EN EL TERREMOTO DE 1985 EN LA CIUDAD DE MÉXICO]

I: y me voy a la / a tientas a la escalera y ca- / y había otros tratando de abrirla / y no podían por lo mismo / total entre todos la abrimos y caían / muros / y caían / vidrios / llovía así / aquí así caían caían caían / entonces queríamos salir / y pos no nos aniba- / animábamos

E: $\operatorname{mjm}$

I: porque / moviéndose todo y cayendo / escombro

$\mathrm{E}:$ ¿pedazos muy grandes?

I: muros completos / total ya le / se para / y este / y descanso obviamente / justo

me puse un pantalón / y bajar la escalera / y como se había roto / y había quedado

la escalera / pues a lo mejor a la altura de la / teja / no sé

E: mjm

I: $\quad$ pos había que saltar $\langle$ risas $=\ll \mathrm{I} » /\rangle /$ pos a saltar descalzo $/$ sobre vidrios $\mathrm{y}$ escombro

E: ¡agh!

I: afortunadamente sólo me herí poquito en los pies

E: $\operatorname{mjm}$

I: con los vidrios / y ya me fui a / a Reforma / contemplar aquello ahí / pesado /

tremendo / muertos y // y ahí tuve / ahí ahí sí la vi muy cerquita

E: ¿cuánto tiempo estuvo ahí después del / del terremoto?

\subsubsection{Intensificación semántica}

Asimismo, el uso de nuestro par adverbial como elemento estratégico de matización, según anunciábamos más arriba, puede también propiciar un efecto pragmático de intensificación. Cuando lo esperable, lo deseable o lo bueno desde la perspectiva del hablante es que algo sea mucho, la introducción de solo-solamente intensifica la negatividad de lo poco o lo pequeño, de nuevo con la importante presencia del factor de la contraexpectativa, del carácter antiorientado del contenido introducido por el adverbio. 
En el ejemplo 11, vemos cómo el informante esperaba que todos los invitados comieran pulpos, pero de diez personas, solo dos lo hicieron y esa implicación se rescata del entorno cognoscitivo compartido del que gozan los interlocutores. Así, si se cocina algo para alguien, lo esperado, lo deseable es que los invitados coman lo que se preparó para ellos, hecho que no sucede en este caso; de ahí el deseo del hablante de mostrar su contrariedad con la situación, que le lleva a intensificar lo poco. Es decir, al hecho objetivo de que dos de las diez personas comieron pulpo se añade la matización intensificadora de que fueron solamente dos las que lo hicieron. Queda claro que, frente a la expectativa de lo mucho, lo poco se considera claramente negativo.

(11) [INFORMANTE CUENTA SU EXPERIENCIA CON INVITADOS CUANDO COCINÓ PULPOS] E: / ¿y las costumbres / cómo son / en / no sé / en una celebración de navidad en Reynosa y / de aquí de Monterrey? / ¿varía mucho?

I: son parecidas / acuérdate que // como decía Vasconcelos / que a mi comadre Lidia le // le clavó una espina en el corazón / donde empieza la carne asada se acabó la cultura $\langle$ risas $=« \mathrm{E} » /\rangle / /$ nunca me le he perdonado / una vez que los invité a cenar a tu casa / y di pulpos / de diez gentes / solamente dos / comieron los pulpos los demás me lo dejaron / entre ellos mi comadre / inculta analfabeta! no te puedo decir porque sí sabes leer $\langle$ risas $=\langle$ todos $» />/$

En el ejemplo 12, de manera similar, se implica que el hablante esperaría que, si el coste de una caja de pastillas es elevado, el contenido fuera mayor de dos unidades. Así, podemos apreciar cómo, de la mano de la contraexpectativa sobre una cantidad mayor, vuelve a aparecer la disconformidad intensificadora de lo poco.

(12) [INFORMANTE EJEMPLIFICA CON UNA EXPERIENCIA EL EXCESIVO COSTO DE LOS MEDICAMENTOS]

I: [y hay algunos]/y hay algunos que valen/ iiciento y pico!!

E: ihh

I: y no creas que traen muchas pastillas

E: sí/ unas [cuatro/ ¿no? (risa)]

I: [traen dieciséis/ no]/// la habitualmente eh < eh:> la mayoría// mínimo traen diez/// eh < eh:>/ un promedio de dieciséis/ y otras traen veinte exagerando/ no traen más

E: ¡jí!/ yo el otro día $\mathrm{mm} / /$ no me acuerdo qué me enfermé/ y me recetaron un antibiótico bastante fuerte/ y venían sólo ¡dos pastillas! en el/ en la cajita y costaba trescientos pesos/ la medicina// $\mathrm{mmhh} /$ dije «híjole»

I: sí/// no pues < pus> sí hay medicamentos que te vale doscientos y pico/ una sola ampolleta

$\mathrm{E}:[\mathrm{mh}]$

I: [nada más]/y párale de contar

E: híjole 
La cuestión, una vez más, parece clara: no se trata únicamente de que la caja traiga pocas pastillas; se trata, desde la óptica del hablante, de que, dado el precio del medicamento, lo esperable, lo deseable, lo bueno sería que trajera muchas pastillas, expectativa frente a la que se introduce el elemento antiorientado solo dos pastillas.

\subsection{Matización ilocutiva}

En el caso de la matización semántica, como hemos observado, la toma de postura (atenuadora o intensificadora) del hablante tiene que ver con el significado de su enunciado, referido a unos hechos cuya representación minimiza o intensifica. Frente a ella, nos hallamos ahora ante un caso distinto, en la medida en que la matización adquiere una naturaleza interactiva, es decir, en cuanto se relaciona directamente con el proceso dialógico y atenúa o intensifica el valor ilocutivo del enunciado del hablante, hecho que la relaciona con la vertiente social del acto comunicativo, es decir, con la cortesía. Esto es, en la medida en que un determinado acto de habla, en cierto contexto, pueda provocar un efecto (des)cortés, la acción atenuadora o intensificadora de nuestro adverbio puede minimizar o potenciar dicho efecto ${ }^{9}$. Por lo demás, se mantiene la misma idea de antiorientación y de contraexpectativa, de manera que el efecto de atenuación o intensificación es fruto del contraste que la reducción conceptualizadora de solosolamente lleva a cabo frente al contexto anterior.

\subsubsection{Atenuación ilocutiva}

En la atenuación ilocutiva, el hablante pretende poner de relieve ante su interlocutor que el acto de habla que está profiriendo no es lo que aparenta o pudiera aparentar, sino otra cosa, en este caso, más suave o sutil. De este modo, frente a posibles conceptualizaciones alternativas de ilocución que pueden estarse llevando a cabo (que pueden estar en el contexto, explícitas o implícitas), el hablante restringe el alcance ilocutivo de sus palabras, y lo hace conforme a motivaciones sociales de cortesía.

En el ejemplo 13, el profesor dice a su estudiante no te estoy criticando, no te estoy insultando... solo digo que; así, restringe la fuerza de su acto de

\footnotetext{
9 Conviene dejar claro que, a diferencia de lo que sostenían algunos de los planteamientos clásicos sobre la cortesía (como Leech 1983), en absoluto partimos de la base de que ciertos actos de habla sean, per se, corteses o descorteses. Al contrario, como discutimos en Fernández García (2016), la fuerza (des)cortés de las enunciaciones, sin carecer de un cierto componente convencional, posee mucho de contextual.
} 
habla, mostrándolo como algo más suave y provocando, de este modo, un efecto cortés ${ }^{10}$.

(13) [PROFESOR CUENTA QUE UNO DE SUS ESTUDIANTES LE RECLAMÓ POR CORREGIR SUS FALTAS ORTOGRÁFICAS EN EL EXAMEN]

I: menos recetar / o diagnosticar / ahora / si te das cuenta en estas partes donde están / revisados con rojo las faltas de ortografía / al final de cuentas ¿la respuesta está bien o está mal? / bueno / está bien / no / si yo te hubiera / dejado sin revisar / la respuesta / por una falta ortográfica / critica mi trabajo / pero si solamente / estoy diciendo / aquí va una uve aquí es ese ce o aquí va con hache / tómalo como un valor agregado a mi trabajo / se fue contenta la persona / años después me lo encuentro en la calle // y dice / profesor // ¿'se acuerda de mí?

Ya sea frente a palabras previas del estudiante en este sentido, ya sea frente a una interpretación que pueda flotar en el ambiente, el profesor restringe el valor ilocutivo de su intervención: no está criticando o despreciando el trabajo, solamente está diciendo..., es decir, está evaluando ecuánimemente ciertos componentes del texto. Es claro, en este sentido, que la atenuación, a diferencia de lo que ocurría en su variante semántica, tiene un valor interactivo, dirigido a rebajar la posible conflictividad del intercambio comunicativo.

Asimismo, en el ejemplo 14, el hablante da su punto de vista sobre cómo es tratada la homosexualidad en una obra teatral; y, de manera explícita, manifiesta su desacuerdo. Sin embargo, vemos que introduce estratégicamente el adverbio solamente para reducir el impacto que su comentario pueda causar en el interlocutor.

(14) [INFORMANTE DA SU OPINIÓN SOBRE CÓMO EL DIRECTOR DE UNA OBRA DE TEATRO TRATA EL TEMA DE LA HOMOSEXUALIDAD]

E: y qué reto/ ¿no?/ porque/ bueno/ ya no me acuerdo bien/ si tenían algún encuentro

I: $[\mathrm{mm}]$

E: [pero]

I: nada más caricias/ finalmente [se ven]

E: [ajá]

I: dentro de la// de la/ de la obra/ nada/ nada/ nada grotesco// pero/ vamos/ eh/// el gusto de la gente/ de alguna manera/ manifestarse/ ¿no?/ $\mathrm{mm} /$ en este caso pues < pus>/ a lo mejor// pudiéramos pensar que el director estaba manifestando su

${ }^{10}$ No entraremos aquí a fondo en el análisis de dicho efecto desde el punto de vista de las teorías de la cortesía. Señalemos, no obstante, que, desde la perspectiva del modelo de la gestión interrelacional de Spencer-Oatey (2002, 2008), dicho efecto cortés puede estar dirigido a diferentes esferas de la figura del interlocutor, como su imagen (cuando se atenúa una aparente crítica, por ejemplo) o sus derechos de socialización (en casos como el de atenuar una aparente orden). 
$\mathrm{E}:[($ risa $)]$

I: [su placer] / o su gusto ¿no? de/ de la ho-/ homosexualidad vivida o/ no sé/ o/ a lo mejor una manera de manifestar que/ la gente/ acepte

E: proyectar/ ¿no?/ [eso]

I: [sí] la/ la homosexualidad/ pero bueno/ yo no voy en contra/ ni a favor/ solamente no estoy de acue-/ no no estoy yo de acuerdo/ y ya (clic)/// así es entonces dentro de la vida cómo/ cómo se van hilando las cosas

Como vemos, por tanto, en este tipo de ejemplos, a diferencia de lo que ocurría en la atenuación del plano semántico, no se pretende minimizar la percepción de determinados hechos externos al discurso (como en solo se resbaló y se cortó, del ejemplo 9, o en solo me herí un poquito los pies, del ejemplo 10), sino que se procura atenuar la percepción del propio discurso, de la carga ilocutiva que el hablante le imprime.

\subsubsection{Intensificación ilocutiva}

Como quedó señalado más arriba, conforme al contenido semántico de nuestro par adverbial, la matización atenuadora parece la más natural. No obstante, tal y como vimos en 4.1.2 en relación con el plano semántico, según cuáles sean las expectivativas y el contexto de enunciación, la reducción antiorientada que provoca puede generar una intensificación de la negatividad de lo expresado por parte del hablante.

Y algo semejante ocurre en el plano ilocutivo. Hablamos, en todo caso, de una restricción en la conceptualización del acto ilocutivo que se lleva a cabo; ahora bien, dicha restricción podrá ir encaminada a:

- mostrar, según veíamos en 4.2.1, como más suave o neutra la posición conversacional del hablante, con un efecto atenuador de perfil cortés; o bien, al contrario,

- mostrar la enunciación como más dura o crítica, intensificada con efecto descortés, en la medida en que las cosas que podrían decirse sobre el interlocutor se reducen a algo malo, excluyendo lo bueno.

Hemos de señalar, no obstante, que no hemos hallado en nuestro corpus casos de este tipo, como podría ser, por ejemplo, el elaborado al efecto como 15.

(15) A: No entiendo por qué me hablas así.

B: Porque solo dices estupideces.

Parece claro cómo, en la respuesta de B, la presencia del adverbio intensifica exponencialmente la carga crítica del acto de habla, y con ella su potencial efecto descortés. Y un comentario muy semejante podría hacerse de un caso como 16. 
(16) Yo siempre hago lo mejor para ti, pero tú solo quieres aprovecharte de mí.

Como decíamos, y al igual que en 15, no se trata únicamente de que se critique algo negativo respecto del interlocutor, sino de que la presencia del operador restringe lo que puede decirse de éste a solo eso, circunstancia que dispara el potencial crítico del enunciado.

Ahora bien, el hecho de que hayamos ilustrado este tipo con ejemplos elaborados ad hoc (por su ausencia en nuestro corpus) evidencia la necesidad de seguir investigando sobre este aspecto, es decir, sobre el modo específico en que, más allá de su valor como matizador semántico, el par adverbial solo-solamente puede actuar como matizador en el plano interactivo. Para empezar, porque, como adelantábamos más arriba y explicábamos en otro lugar (Fernández García 2016: 82-83) conforme a planteamientos de Briz (2012), atenuación y cortesía, por un lado, e intensificación y descortesía, por otro, por más que sean pareja, no pasan de ser pareja de conveniencia y en cualquier momento pueden dejar de serlo. Así, no es difícil imaginar el ejemplo 17, configurado de manera inversa:

(17) Yo siempre me porto mal contigo, pero tú solo haces cosas buenas por mí.

Obsérvese, no obstante, que no se trata de un contraejemplo a nuestro planteamiento de atenuación/intensificación ilocutiva frente a atenuación/intensificación semántica. De hecho, 17, al igual que 16, es un claro ejemplo de intensificación ilocutiva, en el que, frente a las expectativas generadas por la primera parte del enunciado, el adverbio potencia de forma manifiesta una interpretación diametralmente opuesta. La clave, en el caso 17, son la negatividad del contexto y el hecho de que, frente a ella, lo que intensifica el adverbio es un acto de habla que promueve la solidaridad y la cortesía.

\section{Conclusiones}

Partía este trabajo de la constatación, por parte de la bibliografía especializada, de la capacidad del par adverbial solo-solamente para, más allá de las variantes de significado a las que puede estar asociado (y en paralelo a ellas), ejercer una función estratégica de matización, bien de naturaleza atenuadora, bien de naturaleza intensificadora.

Conforme a los objetivos planteados al comienzo, hemos podido corroborar que, efectivamente, el par adverbial solo-solamente, amén del valor significativo básico que desarrolle en cada caso, es utilizado por el hablante para matizar pragmáticamente (atenuándolo o intensificándolo) el conte- 
nido de sus enunciaciones. Ahora bien, la heterogeneidad de los ejemplos hallados nos ha conducido a aislar la presencia de dos tipos diferentes de matización, radicados en el plano semántico y en el plano ilocutivo, respectivamente.

En el nivel del significado, solo-solamente, más allá del valor semántico primario que posea en cada caso, sirve al hablante para presentar una determinada información como insignificante o poco importante (atenuación), o -a la inversa- sumamente importante y, por ende, digna de ser magnificada y de no pasar desapercibida (intensificación). En este sentido, se activan escalas de tipo valorativo en las que poco es considerado bueno o, en ciertas circunstancias, malo, como se ha explicado en las secciones 4.1.1 y 4.1.2.

En el nivel de la fuerza ilocutiva, por su parte, el efecto matizador de solosolamente se relaciona con el proceso dialógico y la vertiente social del acto comunicativo, espacio en el que, de nuevo, puede atenuar, ejerciendo una función de cortesía (es decir, de potenciación del vínculo social entre los interlocutores), o bien intensificar, ejerciendo una función de descortesía (es decir, de deterioro deliberado del vínculo social entre los interlocutores).

No obstante, en 4.2.2 poníamos de manifiesto la necesidad de seguir investigando el funcionamiento y la configuración específica de la matización en el nivel ilocutivo, con la intención de arrojar luz, entre otros aspectos, sobre las asistemáticas relaciones entre atenuación y cortesía, por un lado, e intensificación y descortesía, por otro.

Pongamos también de relieve, por último, dos cuestiones adicionales. En primer lugar, el hecho de que el papel de solo-solamente en el surgimiento de los efectos matizadores analizados resulta central, independientemente de la presencia de otros elementos contextuales y discursivos que puedan contribuir en este sentido. En segundo lugar, la importancia de las ideas de contraexpectativa y antiorientación para entender el modo en que nuestro par adverbial activa sus efectos matizadores.

Podemos, pues, concluir este trabajo señalando que hemos corroborado el funcionamiento como matizador pragmático-discursivo del par adverbial solo-solamente, para cuya caracterización hemos propuesto dos criterios definitorios que se cruzan, a saber, matización atenuadora $v$ s. intensificadora y matización semántica vs. ilocutiva. Y, habiendo ofrecido detalles relativos al funcionamiento discursivo de cada uno de los subtipos, hemos dejado abierto a futuras investigaciones el análisis detallado de la matización ilocutiva. 


\section{BIBLIOGRAFÍA}

Anscombre, Jean-Claude y Oswald Ducrot (1994): La argumentación en la lengua, Madrid: Gredos.

ARJONA, Marina (1991): Estudios sintácticos sobre el habla popular mexicana, México: UNAM.

BARRENECHEA, Ana María (1977): «Operadores pragmáticos de actitud oracional: Los adverbios en -mente», en Juan Miguel Lope Blanch (ed.), Estudios sobre el español hablado en las principales ciudades de América, México: UNAM, 313-332.

BRIZ, Antonio (2012): «La (no)atenuación y la (des)cortesía, lo lingüístico y lo social: ¿'son pareja?», en Julio Escamilla Morales y Grandfield Henry Vega (eds.), Miradas multidisciplinares a los fenómenos de cortesía y descortesía en el mundo hispánico, Barranquilla-Estocolmo: Universidad del Atlántico-Programa Edice, 33-75.

Butragueño, Pedro y Yolanda Lastra (2011): Corpus sociolingüístico de la Ciudad de México, Vol. 1, México: Colegio de México.

Cestero, Ana María, Marta Albelda y Antonio Briz (2014): «Estudio coordinado de la "atenuación" en el marco del PRESSEA: propuesta metodológica», en XVII Congreso Internacional Asociación de Lingüistica y Filología de América Latina, Alfal, Paraíba, Brasil <http://www.mundoalfal.org/CDAnaisXVII/trabalhos/ R0091-2.pdf>.

Cestero, Ana María y Rodríguez, Lidia (2014): «PRESSEA-ATENUACión, Guía de Estudio de la atenuación en los corpus PRESSEA» <http://preseea.linguas. net/Portals/0/An\%C3\%A1lisis\%20de\%20la\%20atenuaci\%C3\%B3n.\%20GU\% C3\%8DA\%20de\%20estudio\%20de\%20la\%20atenuaci\%C3\%B3n.pdf>.

EgEA, Esteban Rafael (1979): Los adverbios terminados en -mente en el español contemporáneo, Bogotá: Publicaciones del instituto Caro y Cuervo.

EscANDELL, María Victoria (1993): Introducción a la pragmática, Barcelona: Anthropos/UNED.

FERnÁndez García, Francisco (2015): «El menosprecio y la burla como armas de ataque en el debate electoral. Caracterización funcional y configuración discursiva», Pragmática Sociocultural / Sociocultural Pragmatics 3, 32-58.

- (2016): «Bases teóricas para un estudio transcultural y variacionista de la (des)cortesía», Estudios de Lingüistica, Universidad de Alicante 30, 79-100.

Ferrari, Laura, Mabel Giammatteo e Hilda Albano, (2011): «Operadores de foco: el caso de incluso, hasta, solo y aun», Cuadernos de la ALFAL 3, 30-41 <http:// mundoalfal.org/sites/default/files/revista/03_cuaderno_005.pdf >.

Fuentes Rodríguez, Catalina (2009): Diccionario de conectores y operadores del espanol, Madrid: Arco/Libros.

Fuentes Rodríguez, Catalina y Esperanza R. Alcaide LARA (2007): La argumentación lingüistica y sus medios de expresión, Madrid: Arco/Libros. 
GUTIÉRREZ, Rodrigo (2008): «La identificación de los tópicos y los focos», Nueva Revista de Filología Hispánica LVI/2, 363-401.

GutiÉRREZ, Yurena María (2010): «Sobre foco nomás», en Víctor M. Castel y Liliana Cubo de Severino (eds.), La renovación de la palabra en el bicentenario de la Argentina, Los colores de la mirada lingüistica, Mendoza: Editorial de la Facultad de Filosofia y Letras de la Universidad Nacional de Cuyo, 625-632.

Hartmann, Steven Lee (1999): «Parsing Spanish solo». En Javier Gutiérrez-Rexach y Fernando Martínez Gil (eds.), Advances in Hispanic Linguistics, Papers from the 2nd. Hispanic Linguistics Symposium, Vol. 1, Somerville: Cascadilla Press, 28-41.

Himmelmann, Nikolaus P. y Eva F. Schultze-Berndt (2005): Secondary Predication and Adverbial Modification, The Typology of Depictives, Oxford: Oxford University Press.

Hoeksema, Jack y Frans ZwarTs (1991): «Some Remarks on Focus Adverbs», Journal of Semantics 8, 51-70.

Hummel, Martin (2012): Polifuncionalidad, polisemia y estrategia retórica. Los signos discursivos con base atributiva entre oralidad y escritura, Berlín: Mouton de Gruyter.

Kaul de Marlangeon, Silvia Beatriz (2002): Los adverbios en -mente del español de hoy y su función semántica de cuantificación, Madrid: Vervuert.

Kovacci, Ofelia (2000): «El adverbio», en Ignacio Bosque y Violeta Demonte (eds.), Gramática descriptiva de la lengua española, 1, Madrid: Espasa, 705-786.

LANGaCker, Ronald W. (1991): Concept, Image, and Symbol: The Cognitive Basis of Grammar, Berlín: Mouton de Gruyter.

- (2000): «Estructura de la cláusula en la gramática cognoscitiva», Revista Espanola de Lingüística Aplicada, vol. extra 1, 19-66.

LARA, Luis Fernando (2011): Diccionario del español de México, México: El Colegio de México.

Lastra, Yolanda y Pedro Butragueño (2000): El modo de vida como factor sociolingüístico en la ciudad de México, México: El Colegio de México.

— y - (2003): Metodología del «Proyecto para el estudio sociolingüístico del español de España y de América», México: El Colegio de México.

- y - (2009): Corpus sociolingüistico de la ciudad de México, Materiales de PRESEEA MEXICO, México: El Colegio de México.

LEech, Geoffrey N. (1983): Principles of Pragmatics, Londres: Longman.

MARTíNEZ, Juan (2006): «Los adverbios en -mente y los diccionarios modernos del español: perspectiva pragmático-discursiva». En Manuel Casado Velarde, Ramón González Ruiz y María Victoria Romero Gualda (eds.), Análisis del discurso: lengua, cultura y valores. Actas del I Congreso Internacional, vol. II, Madrid: Arco/Libros, 1433-1444.

Medina, Lorena Y. y Eva Patricia VeLÁsQuez (2016): «Análisis prosódico del par adverbial solo-solamente en el registro oral-informal del español de Monterrey», Cuadernos de Lingüistica Hispánica 28, 41-67.

Mey, Jacob L. (1979): Pragmalinguistics: Theory and Practice, La Haya: Mouton.

PorTOlÉs, José (1993): «La distinción entre los conectores y otros marcadores del discurso en español», Verba 20, 141-170. 
Real Academia Española y Asociación de Academias de la lengua Española (2010): Manual de la nueva gramática de la lengua española, Madrid: Espasa.

Rodríguez Alfano, Lidia, María Eugenia Flores y Tzitel Pérez (2012): Corpus Monterrey-PRESEEA, México: UANL.

RODRÍGUEZ, R. T. M. (2003): La gramática de los adverbios en -mente o cómo expresar maneras, opiniones y actitudes a través de la lengua, Madrid: Universidad Autónoma de Madrid.

SECO, Manuel (2002): Diccionario de dudas y dificultades de la lengua española, Madrid: Espasa.

SPENCER-OATEY, Helen (2002): «Managing rapport in talk: Using rapport sensitive incidents to explore the motivational concerns underlying the management of relations», Journal of Pragmatics 34, 529-545.

- (2008): «Face, (Im) Politeness and Rapport», en Helen Spencer-Oatey (ed.), Culturally Speaking, Culture, Communication and Politeness Theory, Londres / Nueva York: Continuum, 11-47.

Vigueras, Alejandra (1983): «Sintaxis de los adverbios en -mente en el habla culta de la ciudad de México», Anuario de Letras. Lingüistica y Filología 21, 119-145.

ZubizARReTA, María Luisa (2000): «Las funciones informativas: Tema y foco», en Ignacio Bosque y Violeta Demonte (eds.), Gramática descriptiva de la lengua española, 3, Madrid: Espasa, 4217-4243. 